\title{
15. COOLING OF WHITE DWARFS
}

\author{
H. M. VAN HORN \\ Dept. of Physics and Astronomy and C. E. Kenneth Mees Observatory \\ University of Rochester, Rochester, N.Y., U.S.A.
}

\begin{abstract}
A knowledge of the precise relationship between the age and luminosity of a white dwarf can in principle be used to determine the compositions of the white dwarfs in galactic clusters. To this end the assumptions in Mestel's theory of white dwarf cooling are critically reviewed, and the results of recent work aimed at relaxing these restrictions are briefly summarized. It is concluded that on the basis of current knowledge an accuracy of the order of 10 or $20 \%$ in the ageluminosity relation should be attainable.
\end{abstract}

\section{Introduction}

The theory that the luminosity of a white dwarf is derived from the thermal energy content of the ions in its interior was proposed by Mestel (1952). In this paper Mestel establishes two important points: (1) that the relation between the luminosity $L$ of a white dwarf and the cooling time $t$ is $t \propto L^{-5 / 7}$, for Kramers' law opacity in the envelope, and (2) that the theoretical age of a white dwarf is inversely proportional to the mean atomic weight $A$ of the ions in its interior. For faint white dwarfs such as van Maanen 2 this leads to the conclusion that $A$ must be of the order of 10 in order that the age of the star be less than the age of the Galaxy. The approximations in the theory, however, preclude the possibility of distinguishing between $A=4$ and $A=24$. Consequently, it has not yet been possible to determine from the observational data what phase (or phases) of nuclear burning occur immediately prior to evolution to the white dwarf stage.

It is the aim of this review to show that recent advances have brought the theory of white dwarf evolution to the stage where theoretical ages with an uncertainty of the order of tens of percents, rather than factors of two, are now possible. With accuracies of this sort, comparison of the theoretical age-luminosity relation with the known ages of the galactic clusters can provide reliable determinations of the mean chemical compositions of the cluster white dwarfs. Similarly, the shape of the observed white dwarf luminosity function, which is simply related to the age-luminosity relation and is primarily affected by the thermal structure of the envelope, may lead to important new conclusions about the outer layers of the white dwarfs.

Since a number of excellent reviews (Schwarzschild, 1958, Chapter 7; Mestel, 1965; Weidemann, 1968) have dealt with various aspects of Mestel's original theory, I shall mainly restrict myself to discussions of the newer developments in the theory of white dwarf cooling. It is convenient for this purpose to begin with a critical summary of the Mestel theory, with particular emphasis on those approximations that have been shown to lead to uncertainties of the order of a factor of two in the theoretical ageluminosity relation. 


\section{Critical Review of Mestel's Theory}

The luminosity of a star is in general given by (see, e.g., Hayashi et al., 1962, p. 39)

$$
L=\int_{0}^{M}\left(\varepsilon-T \frac{\partial s}{\partial t}\right) \mathrm{d} M_{r}
$$

where $\varepsilon$ is the rate of energy generation (or loss) per unit mass by nuclear processes, $T \partial s / \partial t$ is the time rate of change of the heat content per unit mass, and $M_{r}$ is the mass interior to radius $r$. Since $s$, the entropy per gram, can be regarded as a function of the temperature $T$ and density $\varrho$ of the stellar matter, we have

$$
T \frac{\partial s}{\partial t}=T\left[\left.\frac{\partial s}{\partial T}\right|_{\varrho} \frac{\partial T}{\partial t}+\left.\frac{\partial s}{\partial \varrho}\right|_{T} \frac{\partial \varrho}{\partial t}\right]=C_{v} \frac{\partial T}{\partial t}-\left.\frac{T}{\varrho^{2}} \frac{\partial P}{\partial T}\right|_{\varrho} \frac{\partial \varrho}{\partial t} .
$$

If nuclear (and neutrino) processes are neglected, following Mestel, (Approximation 1), and if the energy released by residual gravitation contraction is ignored $(\partial \varrho / \partial t=0$ : Approximation 2), the luminosity of a white dwarf is directly proportional to the time rate of decrease of the temperature.

For a non-relativistic degenerate electron gas, the electronic contribution to the specific heat is

$$
C_{v}^{\text {(elect) }}=\frac{3}{2} \frac{k}{A H} \cdot \frac{\pi^{2}}{3} Z \frac{k T}{\varepsilon_{F}}
$$

where $Z, A$ are respectively, the atomic charge and mass, $k=1.38 \times 10^{-16} \mathrm{erg}(\mathrm{K})^{-1}$ is Boltzmann's constant, and $H=1.66044 \times 10^{-24} \mathrm{~g}$ is the unit of atomic mass. Because of the high degeneracy $k T$ is much less than the Fermi energy

$$
\varepsilon_{F}=\frac{\left(3 \pi^{2}\right)^{2 / 3}}{2} \frac{\hbar^{2}}{m_{e}}\left(\begin{array}{c}
\varrho \\
\mu_{e} H
\end{array}\right)^{2 / 3}
$$

of the electrons, and $C_{v}^{(\text {elect })}$ is ignored in comparison with the specific heat of the ions (Approximation 3), which for a non-interacting ion gas becomes (Approximation 4)

$$
C_{v}^{(\text {ion })}=\frac{3}{2} \frac{k}{A H}
$$

The high degeneracy in the core of a white dwarf also promotes highly efficient heat conduction by the degenerate electrons, as was first shown by Marshak (1940). The core is therefore very nearly isothermal (Approximation 5), so that one finally obtains

$$
L \approx-\frac{3 k M \partial T_{c}}{2 A H \partial t},
$$

where $T_{c}$ is the core temperature. 
In order to calculate $T_{c}$, one must deal with the problem of heat transfer through the thin, non-degenerate envelope of the white dwarf. If the envelope is in radiative equilibrium (Approximation 6), and if Kramers' law, $K=K_{0} \varrho T^{-3,5}$ is used to represent the opacity (Approximation 7), the envelope equations can be integrated analytically, giving for the 'radiative, zero' surface conditions, $P=0$ at $T=0$, the result (Schwarzschild, 1958, p. 91)

$$
\frac{1}{8.5} T^{8.5}=\frac{3}{4 a c} K_{0} \frac{\mu H}{k} \frac{L}{4 \pi G M} \frac{1}{2} P^{2},
$$

where $\mu$ is the mean molecular weight of the envelope and $P$ is the pressure. Since at the boundary of the isothermal, degenerate core the pressure and temperature are related through the condition $k T \approx \varepsilon_{F}$ (or $\varrho / \mu_{e} \approx 2.4 \times 10^{-8} T^{3 / 2}$ : Schwarzschild, 1958, p. 60), Equation (6) can be reduced to a relation between core temperature and luminosity:

$$
{ }_{L_{\odot}}^{L} \approx 1.7 \times 10^{-3}{ }_{M_{\odot}}^{M}\left(\frac{4 \times 10^{23}}{K_{0}}\right){ }_{\mu_{e}^{2}}^{\mu} T_{c, 7}^{3,5},
$$

where $\mu_{e}=A / Z$ is the mean molecular weight per electron, and $T_{c, 7}$ is the core temperature in units of $10^{7} \mathrm{~K}$. With the aid of the result (7), Equation (5) can be integrated directly to give the age-luminosity law

$$
\tau \approx \frac{7.6 \times 10^{7}}{A}\left(\frac{K_{0}}{4 \times 10^{23}} \frac{\mu_{e}^{2}}{\mu}\right)^{2 / 7}\left(\begin{array}{c}
M \\
M_{\odot}
\end{array}\right)^{5 / 7}\left(\begin{array}{c}
L \\
L_{\odot}
\end{array}\right)^{-5 / 7} y .
$$

Equations (7) and (8) contain the main results of Mestel's theory. The principal approximations of the theory are summarized in Table $I$, and we next turn to a detailed investigation of the validity of these assumptions.

TABLE I

Main approximations in Mestel's model

1. Neglect of nuclear energy sources and sinks.

2. $T_{\partial t}^{\partial s} \approx C_{r} \frac{\partial T}{\partial t}, \frac{\partial \varrho}{\partial t}=0$ : Neglect of residual gravitational contraction.

3. $C_{v} \approx C_{v}{ }^{(\text {ion })}:$ Neglect of electronic heat capacity.

4. $C_{v}{ }^{(\text {ion })} \approx \frac{3}{2} \frac{k}{A H}$ : Use of perfect gas law for ions.

5. $T(r) \approx T(0) \equiv T_{c}$ : Isothermal core approximation.

6. Assumption of radiative equilibrium in envelope.

7. Use of Kramers' law opacity in envelope.

\section{Nuclear and Neutrino Processes}

\section{A. NUCLEAR ENERGY PRODUCTION}

Under conditions typical of the interior of a white dwarf $\left(\varrho \sim 10^{6} \mathrm{~g} \cdot \mathrm{cm}^{-3}, T \sim 10^{7} \mathrm{~K}\right)$ hydrogen-burning nuclear reactions generate energy at an enormous rate, and - if the 
hydrogen abundance is at all appreciable - generate far more energy than is required to maintain the luminosity of the star. The condition that the total rate of energy production not exceed the observed white dwarf luminosity is alone sufficient to show $X_{H}<10^{-4}$ (Marshak, 1940). For normal white dwarfs, however, much more stringent limitations are set by the following stability considerations (Schatzman, 1958; Schwarzschild, 1958; Mestel, 1965):

Suppose first that nuclear energy production occurs near the base of the nondegenerate envelope. As shown by Ledoux and Sauvenier-Goffin (1950), this situation leads to pulsational instability. For most white dwarfs this possibility is therefore ruled out as a significant contributor to the luminosity by the negative results of the recent observational search for pulsations in degenerate stars by Ostriker and his colleagues (Lawrence et al., 1967). Nuclear burning must thus take place, if at all, in the deep interior of a white dwarf, where it cannot drive pulsations. However, for white dwarfs with $M \gtrsim 0.1 M_{\odot}$ (the approximate lower mass limit for the hydrogenburning main sequence), all hydrogen in the deep interior must already have been consumed. Furthermore, if some residual hydrogen did remain, any appreciable nuclear burning in the degenerate core would result in a thermal runaway, since a rise in the temperature is not offset by a proportionate increase in the pressure in degenerate matter, and the resulting secularly unstable situation is not of interest to a study of stable white dwarfs.

It appears, then, that nuclear reactions are unimportant for 'normal' white dwarfs, and I shall not discuss them further.

\section{B. NEUTRINO ENERGY LOSSES}

Adams et al. (1963) and Inman and Ruderman (1964), have shown that decay of a photon (or 'transverse plasmon') into a neutrino-antineutrino pair becomes possible in the dense plasma of the interiors of stars in the later phases of stellar evolution where $\hbar \omega_{p} / k T \sim 1$. Here $\omega_{p}=\left[4 \pi n_{e} e^{2} / m_{e}\right]^{1 / 2}$ is the electron plasma frequency. This condition is satisfied in the immediate pre-white-dwarf evolutionary stages. Vila (1965) has studied the effect of this energy loss mechanism upon the evolution of a $1 M_{\odot}$ pure iron star. More recently similar calculations have been done for iron stars of other masses by Savedoff et al. (1969), for carbon stars by Beaudet and Salpeter (1969) and by Kutter and Savedoff (1969), and for stars composed of $80 \% \mathrm{O}, 10 \% \mathrm{Ne}$, $10 \% \mathrm{Mg}$ by Vila $(1966,1967)$.

The effect of the plasma neutrino energy loss upon the evolution of a star in the immediate pre-white-dwarf stages is to deplete the thermal energy store on a timescale $\tau_{v} \sim k T / A H \varepsilon_{v}$, where $\varepsilon_{v}$ is the energy loss rate in $\mathrm{erg} \mathrm{gm}^{-1} \mathrm{sec}^{-1}$. At $T \sim 10^{8} \mathrm{~K}$, $\varrho \sim 10^{6} \mathrm{~g} \cdot \mathrm{cm}^{-3}$, the more recent, improved calculation of $\varepsilon_{v}$ by Beaudet et al. (1967) show $\tau_{v} \sim 3 \times 10^{7} \mathrm{~A}^{-1} \mathrm{yr}$. This may be compared with the rate of evolution in the absence of neutrino losses at a similar stage, as given by Equation (8): $\tau \sim 5 \times 10^{7} A^{-1} \mathrm{yr}$. The neutrino losses considerably accelerate the evolution in those phases where $\tau_{v} \leqslant \tau$. This is shown graphically for several of the pure iron star models of Savedoff et al. (1969) in Figure 1, where the ages of the stars are given as functions of their optical 
luminosities. At $10^{-2} L_{\odot}$ the neutrino models are younger by about a factor of 10 , while even at $10^{-4} L_{\odot}$ their ages are still $30-40 \%$ less than the ages of the corresponding models computed without neutrino emission. (These calculations used the older neutrino rates of Inman and Ruderman (1964) that were too large by a factor of 4 (Zaidi, 1965).) The age differences computed with the corrected neutrino rates of Beaudet et al. (1967) would be about a factor of 2.5 at $10^{-2} L_{\odot}$ and about $10 \%$ at $10^{-4} L_{\odot}$.) Since the timescale of evolution is proportional to the total thermal

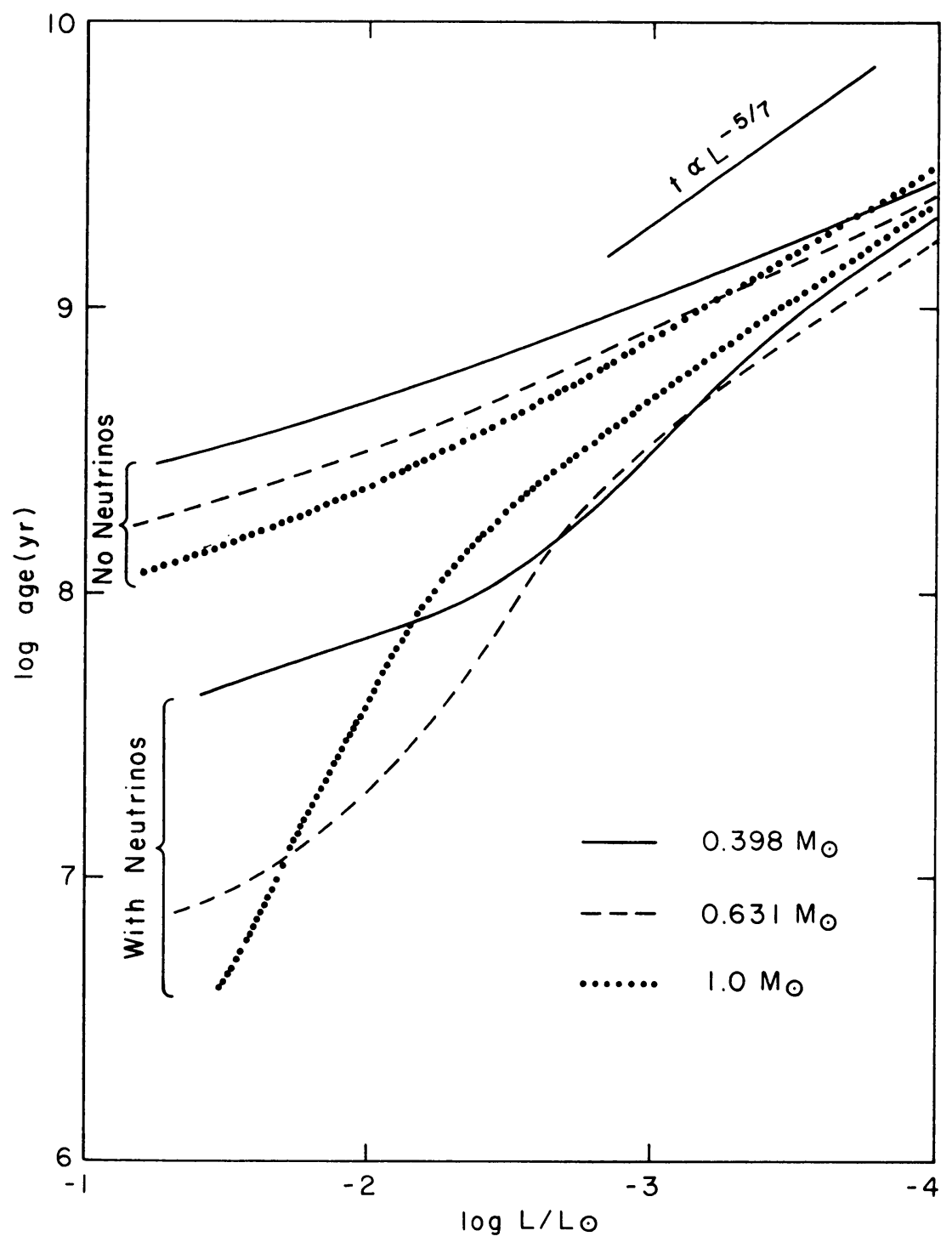

Fig. 1. Effect of plasma neutrino emission on the ages of iron white dwarfs as computed by Savedoff et al. (1969). The slope of the age-luminosity relation $t \propto L^{-5 / 7}$ derived by Mestel (1952) is also shown. 
energy content of 1 white dwarf (and thus to $A^{-1}$ ), no matter whether neutrino or photon emission is the dominant mechanism of energy loss, the ratio of ages of models with the same luminosity but with and without the inclusion of neutrino energy losses is independent of the chemical composition. Thus the effect is important for the brighter white dwarfs of all compositions if the direct electron-neutrino interaction actually does exist in nature, as predicted by Feynman and Gell-Mann (1958), and by Sudarshan and Marshak (1958), and accurate calculations of the ages of the white dwarfs must include the plasma mechanism of neutrino energy loss.

\section{Gravitational Contraction and the Heat Capacity of the Electrons}

The neglect of terms proportional to $k T / \varepsilon_{F}$ in the equation of state of the degenerate electron gas is strictly justifiable only for rather high densities and low temperatures, conditions which are not met in the interiors of all white dwarfs; for example, at the average density of a $0.4 M_{\odot}$ white dwarf $k T / \varepsilon_{F}>0.1$ for $T>2 \times 10^{7} \mathrm{~K}$. The two main consequences of this are that for the less massive white dwarfs it is not sufficiently accurate to neglect either the residual gravitational contraction of the star or the contribution of the electrons to the specific heat of the stellar matter.

The order of magnitude of the contributions of these effects to net rates of energy release in the star can be established in the following way.

It follows from Equation (3) that the electronic contribution to the luminosity of a white dwarf is of the order of $\frac{1}{3} \pi^{2} Z\left(k T / \varepsilon_{F}\right)$ times that of the ions. For reasonable chemical compositions the electrons can even contribute substantially more than the ions, in the hotter white dwarfs. This is shown in Figure 2 for the pure iron models of Savedoff et al. (1969), where the integrals over the entire star of $\varepsilon_{\text {ion }}=C_{v}^{(\text {ion })} \partial T / \partial t$, $\varepsilon_{\text {elect }}=C_{v}^{(\text {elect })} \partial T / \partial t$, and $\varepsilon_{\text {grav }}=-T \varrho^{-2}(\partial P / \partial T)_{\varrho} \partial \varrho / \partial t$ are plotted as functions of the stellar luminosity. It is evident that the approximation of neglect of the electronic heat capacity introduces errors of more than a factor of 2 in the lifetimes of the more luminous iron white dwarfs of all masses, while the effect continues to much lower luminosities for the stars of smaller mass. Because of the dependence of $C_{v}^{(\text {elect })} / C_{v}^{\text {(ion) }}$ on $Z$, the use of an iron composition exaggerates this effect; however, even for a carbon composition, the electrons can still contribute as much as $30-50 \%$ of the luminosity of the hotter white dwarfs.

The contribution of the residual gravitational contraction to the energy balance is much smaller than the effect of the electronic heat capacity. We estimate the magnitude of this term using an argument due to Mestel and Ruderman (1967). They show that for white dwarfs of low mass

$$
\int_{0}^{M} \mathrm{~d} M_{r} \frac{P^{(\text {elect })}}{\varrho^{2}} \Delta \varrho \approx-2 \int_{0}^{M} \mathrm{~d} M_{r} \int_{0}^{T} C_{v}^{(\text {ion })} \mathrm{d} T \equiv-2 E_{\text {therm }},
$$

where $\Delta \varrho$ is the difference in density from the fully degenerate, 'black dwarf' state, 


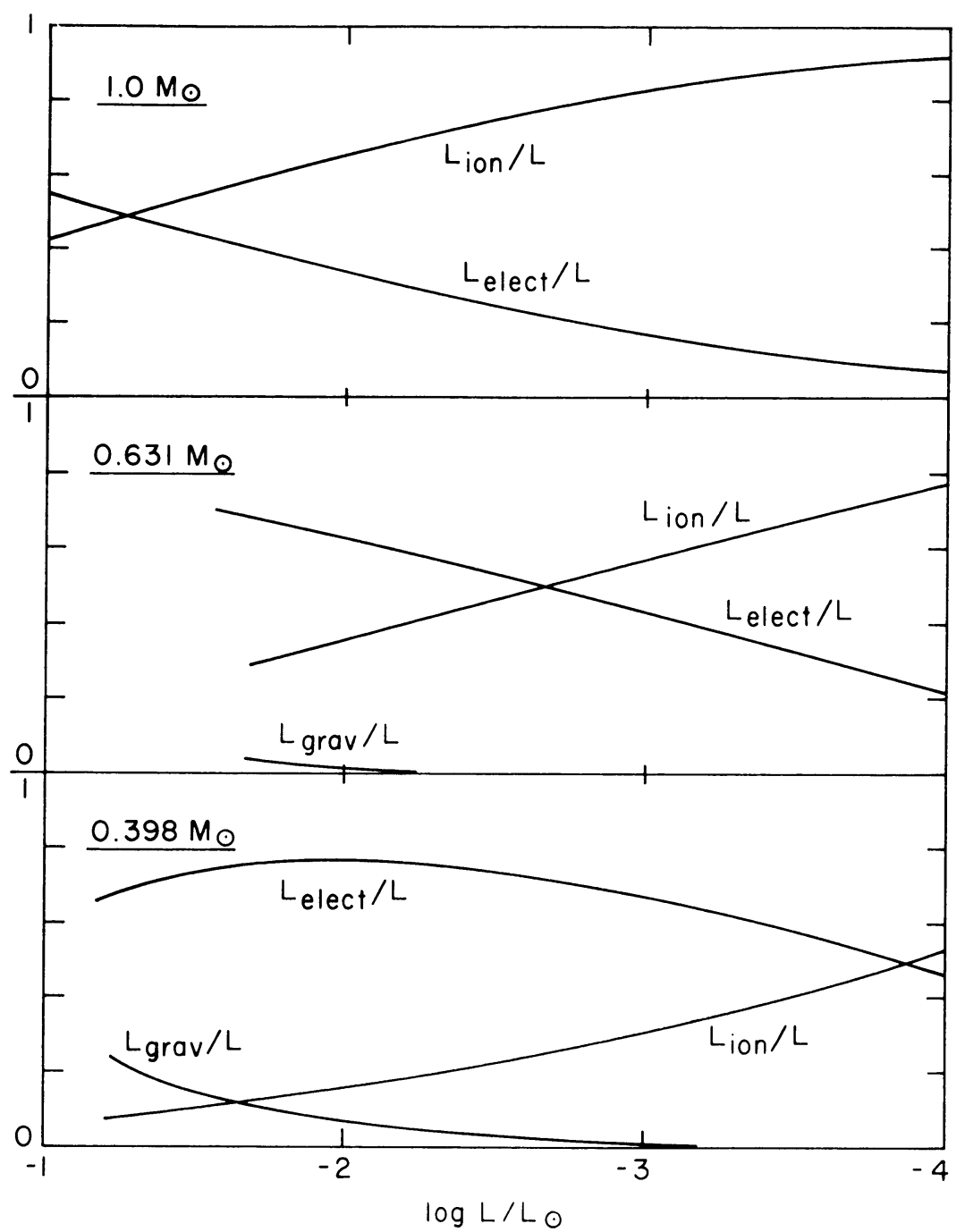

Fig. 2. Contributions of ion cooling, electron cooling and gravitational contraction to the luminosities of the iron white dwarfs without neutrino emission computed by Savedoff et al. (1969).

and we have neglected the coulomb interaction terms (which are discussed in the following section). As shown by Equation (2), the total 'gravitational' energy release is

$$
\Delta E_{\text {grav }}=-\int_{0}^{M} \mathrm{~d} M_{r} \varrho^{2} \frac{\partial P}{\partial T} \varrho_{\varrho} \Delta \varrho=-\int_{0}^{M} \mathrm{~d} M_{r} \frac{P^{(\text {elect })}}{\varrho^{2}} \Delta \varrho\left[\begin{array}{cc}
T & \partial P \\
P^{(\text {elect })} \partial T_{e}
\end{array}\right] .
$$

Since the thermal correction to the degenerate electron pressure is $O\left(k T / \varepsilon_{F}\right)^{2}$, it is 


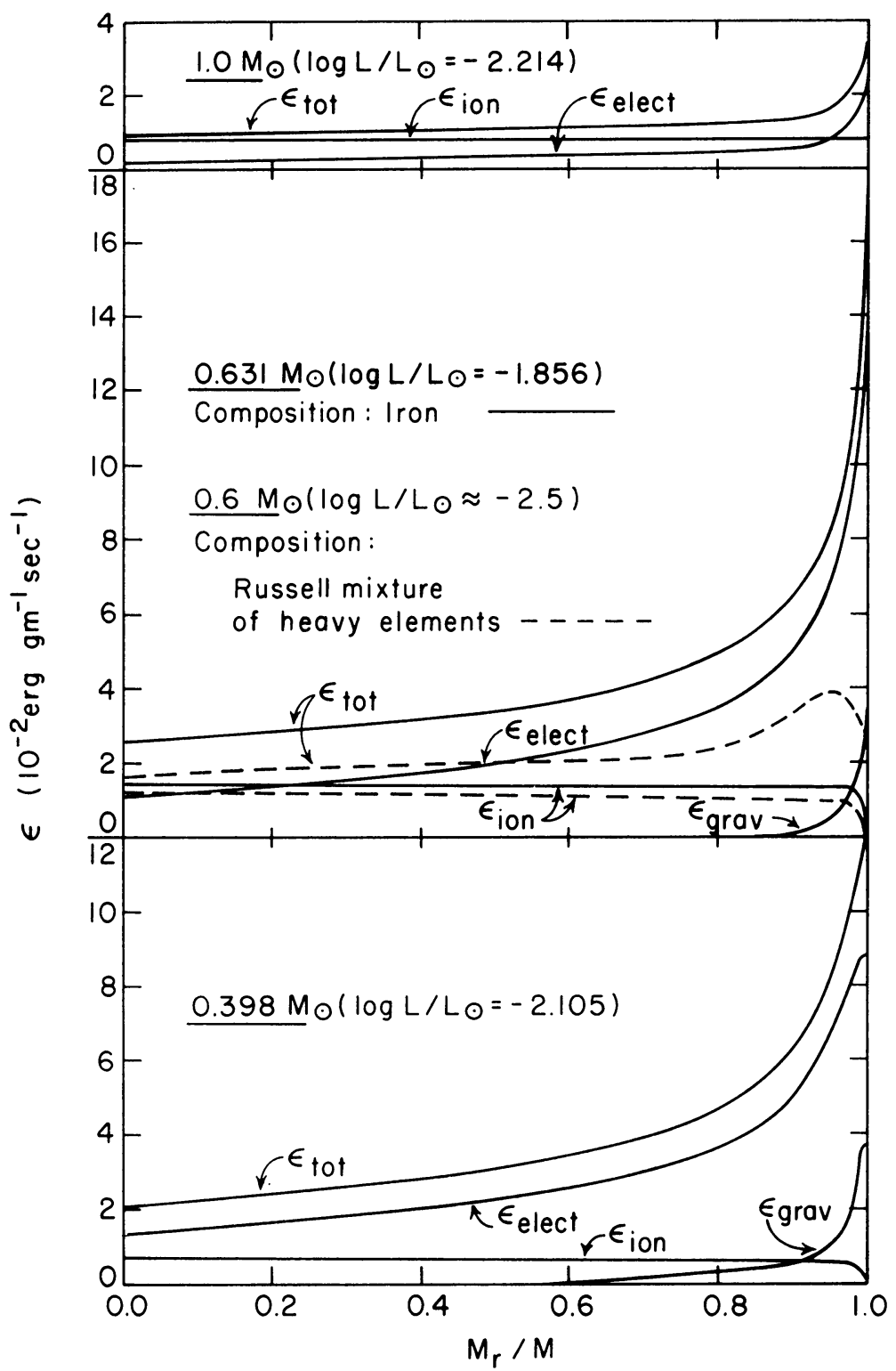

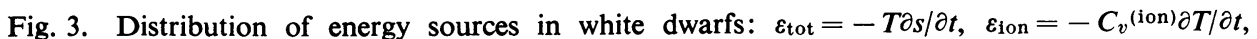
$\varepsilon_{\text {elect }}=-C_{v}{ }^{\text {(elect) }} \partial T / \partial t, \varepsilon_{\mathrm{grav}}=-T Q^{-2}(\partial P / \partial T)_{\varrho} \partial \varrho / \partial t$. The solid curves refer to the iron white dwarf models without neutrino emission computed by Savedoff et al. (1969) and the dashed curves to the $0.6 M_{\odot}$ white dwarf composed of the Russell mixture of heavy elements constructed by

Hayashi et al. (1962). 
negligible, and $\partial P /\left.\partial T\right|_{\varrho} \approx k \varrho / A H$, from the ionic terms alone. Thus we have

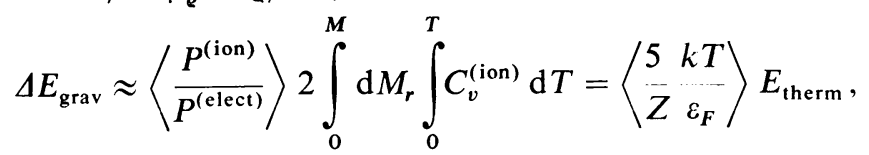

where the average is defined by Equation (10).

The gravitational energy released thus can normally be neglected in a white dwarf, except in a star of very low mass (where $\left\langle k T / \varepsilon_{F}\right\rangle$ may be of the order of $10 \%$ or greater even at typical white dwarf luminosities), and in the outer, non-degenerate envelope. These conclusions are illustrated both by the ratio of the gravitational to total luminosity plotted in Figure 2, and by the distribution of energy sources in white dwarfs of different masses, as shown in Figure 3 for several of the models of Savedoff et al. (1969). Except in the low mass models, the gravitational term is completely negligible. Also shown in this figure is the distribution of energy sources in a $0.6 M_{\odot}$ star composed of the Russell mixture of heavy elements, as computed by Hayashi et al. (1962, p. 164). The agreement between the two results is quite close when the contributions $\varepsilon_{\text {elect }}$ are scaled by the ratio of average ionic charges as indicated by Equation (3).

\section{Effects of Coulomb Interactions}

The recent advances in our understanding of the thermodynamic state of the interior of a white dwarf stem from the recognition - independently by Kirzhnits (1960), Abrikosov (1961), and Salpeter (1961) - that coulomb interactions at the low temperatures characteristic of the white dwarfs force the ions in the plasma to form a crystalline solid. The thermodynamic properties of the stellar plasma in these stages of evolution are dependent upon two dimensionless parameters, the ratio $\Gamma$ of the typical coulomb interaction energy to $k T$ and the ratio of the characteristic energy of lattice vibrations ('phonons') to $k T$. For a plasma containing only one species of ion, these quantities become

$$
\Gamma \equiv \frac{(Z e)^{2}}{a k T}=2.28 \stackrel{Z^{2} \varrho_{6}^{1 / 3}}{A^{1 / 3} T_{7}}
$$

and

$$
\frac{\hbar \Omega_{P}}{k T}=2.240 \frac{\Theta}{T}=2.240 \times 0.174\left(\frac{2 Z}{A}\right) \frac{\varrho_{6}^{1 / 2}}{T_{7}},
$$

where $\varrho_{6}=\varrho / 10^{6} \mathrm{~g} \cdot \mathrm{cm}^{-3}, \frac{4}{3} \pi a^{3}=(\varrho / A H)$ defines the radius $a$ of a sphere which on the average contains one single ion, $\Omega_{p}^{2}=4 \pi(\varrho / A H)\left(Z e^{2}\right) / A H$ is the square of the ion plasma frequency, and $\Theta$ is the Debye temperature of the solid.

These two parameters separate the evolution of a cooling white dwarf naturally into the following four stages*: (i) $\Gamma<\Gamma_{m}, T>\Theta$; in this stage the ions of the plasma

* Mestel and Ruderman (1967), applying the Lindemann melting point rule, found the transition between the high temperature coulomb liquid and the low temperature coulomb solid to occur at $\Gamma=\Gamma_{m} \approx 64$. A somewhat more accurate treatment of this calculation gave $\Gamma_{m} \approx 170$ (Van Horn, 1969). The numerical 'experiments' of Brush et al. (1966) yielded $\Gamma_{m} \approx 125$. 
form a coulomb liquid. (ii) $\Gamma \gtrsim \Gamma_{m}, T>\Theta$; the ions crystallize into a regular lattice structure. (iii) $\Gamma>\Gamma_{m}, T<\Theta$; the specific heat of the lattice decreases rapidly to zero. (iv) $\Gamma<\Gamma_{m}, T<\Theta$; in this case the zero point motion of the ions is sufficient to prevent crystallization, and the final, zero-temperature state of the plasma is that of a charged quantum liquid instead of a solid.

I shall briefly describe the features of each of these regimes and discuss the attendant changes in the rates of evolution of the white dwarfs.

\section{A. COULOMB LIQUID STAGE: $\Gamma<\Gamma_{m}, T>\Theta$}

In Figure 4 the locus of points in the $\mathrm{H}-\mathrm{R}$ diagram where $\Gamma=100$ are shown for white dwarfs composed entirely of ${ }^{4} \mathrm{He},{ }^{16} \mathrm{O}$, or ${ }^{56} \mathrm{Fe}$, together with the observational data. It is apparent that Coulomb effects become extremely important in just the region occupied by the white dwarfs. Also shown in this figure are the 'experimental' data of Brush et al. (1966) for the coulomb corrections to the pressure and entropy of an ideal gas of ions, together with semi-empirical fits to the data which are given by

$$
\begin{aligned}
& \frac{\left(P-P_{0}\right) V}{N k T}=-0.113 \Gamma^{3 / 2}\left[\left(1+\frac{1}{0.142} \Gamma\right)^{1 / 2}+\frac{1.54}{(1+0.575 \Gamma)^{3 / 2}}\right], \\
& \frac{S-S_{0}}{N k}=-\ln \left[1+\frac{\Gamma^{3 / 2}}{2 \sqrt{3}}\left(0.015+\frac{0.585}{1+\Gamma^{1 / 2}}+\frac{0.400}{1+1.308} \Gamma^{3 / 2}\right)\right],
\end{aligned}
$$

where $P_{0}, S_{0}$ are the pressure and entropy of a perfect gas of $\mathrm{N}$ ions in a box of volume $V$ at temperature $T$. These expressions reduce to the correct, analytical results for $\Gamma \ll 1$ and $\Gamma \gg 1$ and are accurate to better than $10 \%$ over the range where the corrections are appreciable.

The coulomb correction terms have two effects upon the lifetimes of the white dwarfs. First, the correction to the entropy leads to a gradual increase in the specific heat from $\frac{3}{2} k / A H$ to $3 k / A H$ as the temperature drops toward the freezing point of the plasma. For the large $\Gamma$ values typical of white dwarfs the heat capacity is very nearly $3 \mathrm{k} / \mathrm{AH}$ over the whole range of interest, and as first pointed out by Mestel and Ruderman (1967) this leads to a factor of two increase in the white dwarf lifetimes during this stage.

The second effect is to increase the rate of gravitational contraction above that indicated by Equation (11). With the aid of Equation (14) we find the contraction rate in the limit of large $\Gamma$ to be about 1.7 times as great as given by Equation (11). For the low mass white dwarfs this effect is therefore not negligible.

B. STAGE OF CRYSTALLIZATION: $\Gamma \gtrsim \Gamma_{m}, T>\Theta$

When the temperature of the white dwarf core falls to the point where $\Gamma=\Gamma_{m}$ at the center, crystallization of the plasma begins. As shown by Brush et al. (1966), a heat of fusion $T \Delta s \sim \frac{3}{4} k T / A H$ is liberated in the transition from the liquid to the solid phase. This release of energy increases the time spent in the corresponding region of the $\mathrm{H}-\mathrm{R}$ diagram by as much as $50 \%$ above the time computed by Mestel and 
COOLING OF WHITE DWARFS



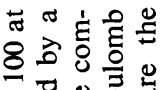

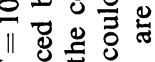

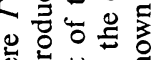

造范的

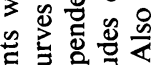

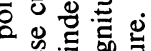

顿.

$\underline{\delta} . \equiv$

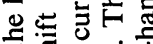



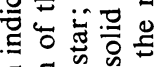

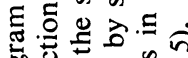

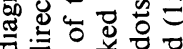

$\simeq$ ช。

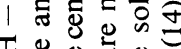

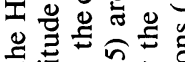

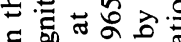

ฐ (1)

ए

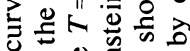

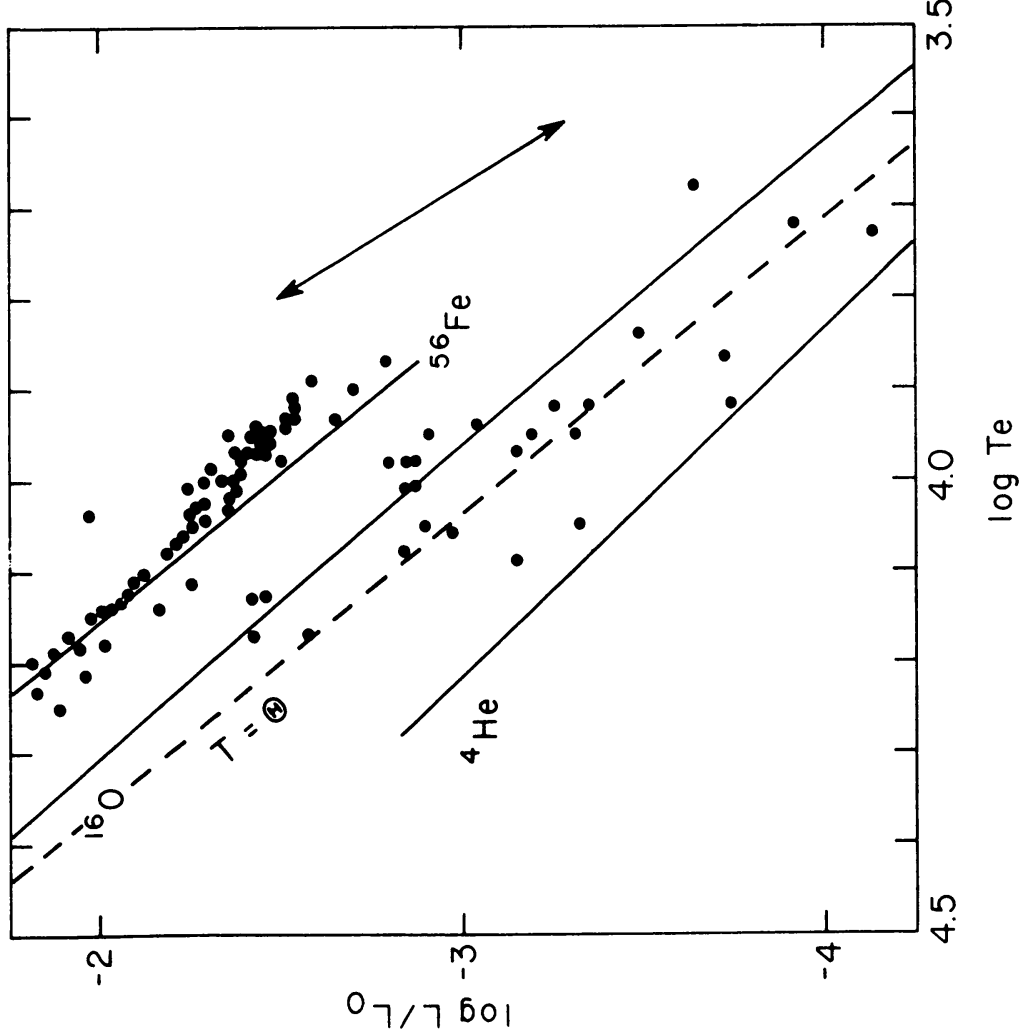

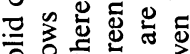

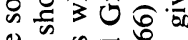

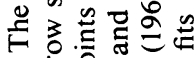

๘



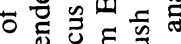

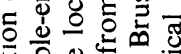

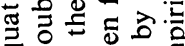

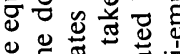

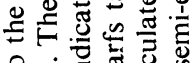

온

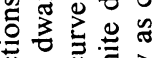

。․ㄹ

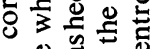

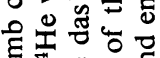

흠 它

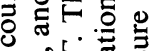

论然

政

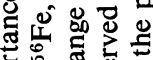

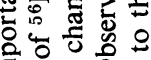

है क त ०

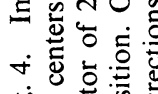

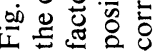


Ruderman (Van Horn, 1968). Because of the strong composition dependence of the Coulomb interaction, as shown, for example, by Equation (12), white dwarfs of different compositions undergo crystallization along different, rather well-defined sequences in the $\mathrm{H}-\mathrm{R}$ diagram. It now appears doubtful, however, whether these sequences can be distinguished observationally (Weideman, 1969).

C. ONSET OF RAPID COOLING: $\Gamma>\Gamma_{m}, T<\Theta$

When $T$ drops below the Debye temperature $\Theta$ of the lattice, excitation of the higher phonon energy levels becomes impossible, and the specific heat begins to fall, decreasing as

$$
C_{v}^{(\text {ion })} \sim \frac{1}{5} 12 \pi^{4}(T / \Theta)^{3} k / A H \quad \text { for } \quad T \ll \Theta
$$

(Landau and Lifshitz, 1958, p. 187ff). The total thermal energy content given by Equation (9) is thus

$$
E_{\text {therm }}=\int_{0}^{M} \mathrm{~d} M_{r} \int_{0}^{T} C_{v}^{(\text {ion })} \mathrm{d} T \rightarrow\left\{\begin{array}{l}
3 k \\
A H \\
3 k \\
\frac{3 H}{A H} T_{c} M \cdot T_{5} \int_{0}^{M} \frac{\mathrm{d} M_{r}}{M}\left(\begin{array}{c}
T_{c} \\
\Theta
\end{array}\right)^{3}, T_{c} \ll \Theta
\end{array}\right.
$$

The density dependence of $\Theta$ (Equation (13)) requires the integration over the entire mass to obtain the heat capacity of the entire star. The results of integrating the full Debye expression for the specific heat over the mass distributions for a number of white dwarfs have been tabulated by Van Horn (1968), and the substantial reduction in the lifetimes of the fainter white dwarfs has been discussed by Mestel and Ruderman (1967), by Van Horn (1968), and by Ostriker and Axel (1969). The latter paper shows that the inclusion of this effect leads to ages $<10^{10} \mathrm{yr}$ for all white dwarfs more massive than about $0.7 M_{\odot}$.

The upper boundary of the region of rapid cooling in the $\mathrm{H}-\mathrm{R}$ diagram is indicated in Figure 4, where the locus of points for which $T=\Theta$ at the center of the star is shown.

D. QUANTUM LIQUID STAGE: $\Gamma<\Gamma_{m}, T<\Theta$

When the temperature falls below $\Theta$ while $\Gamma<\Gamma_{m}$, the zero-point motions of the ions are sufficient to prevent the plasma from crystallizing, and the final, zero-temperature state is then that of a quantum liquid. The exact value of the stellar mass at which this transition occurs depends upon the value of $\Gamma_{m}$ and is smaller for larger $\Gamma_{m}$. Because of the rather limited range of mass and composition for which white dwarfs can possess quantum liquid cores this stage of evolution has been largely neglected. However, see Abrikosov (1961).

\section{Radiative Transfer near the Core-Envelope Boundary}

The thermal structure of a typical white dwarf is shown in Figure 5, where the model of Sirius B computed by Marshak (1940) is given. At the boundary of the degenerate 
core $(\psi=0)$, which is reached at a depth of one percent of the stellar radius, the temperature has already climbed to about half the value at the center of the star. It is at approximately this point also that the change from radiative transport to electron conduction occurs. This is indicated by the run of radiative and conductive opacities also shown in the figure. Since about half of the total temperature rise takes place in the non-degenerate envelope while most of the remaining change occurs in the outer part of the degenerate core, it is important to have accurate knowledge of both the
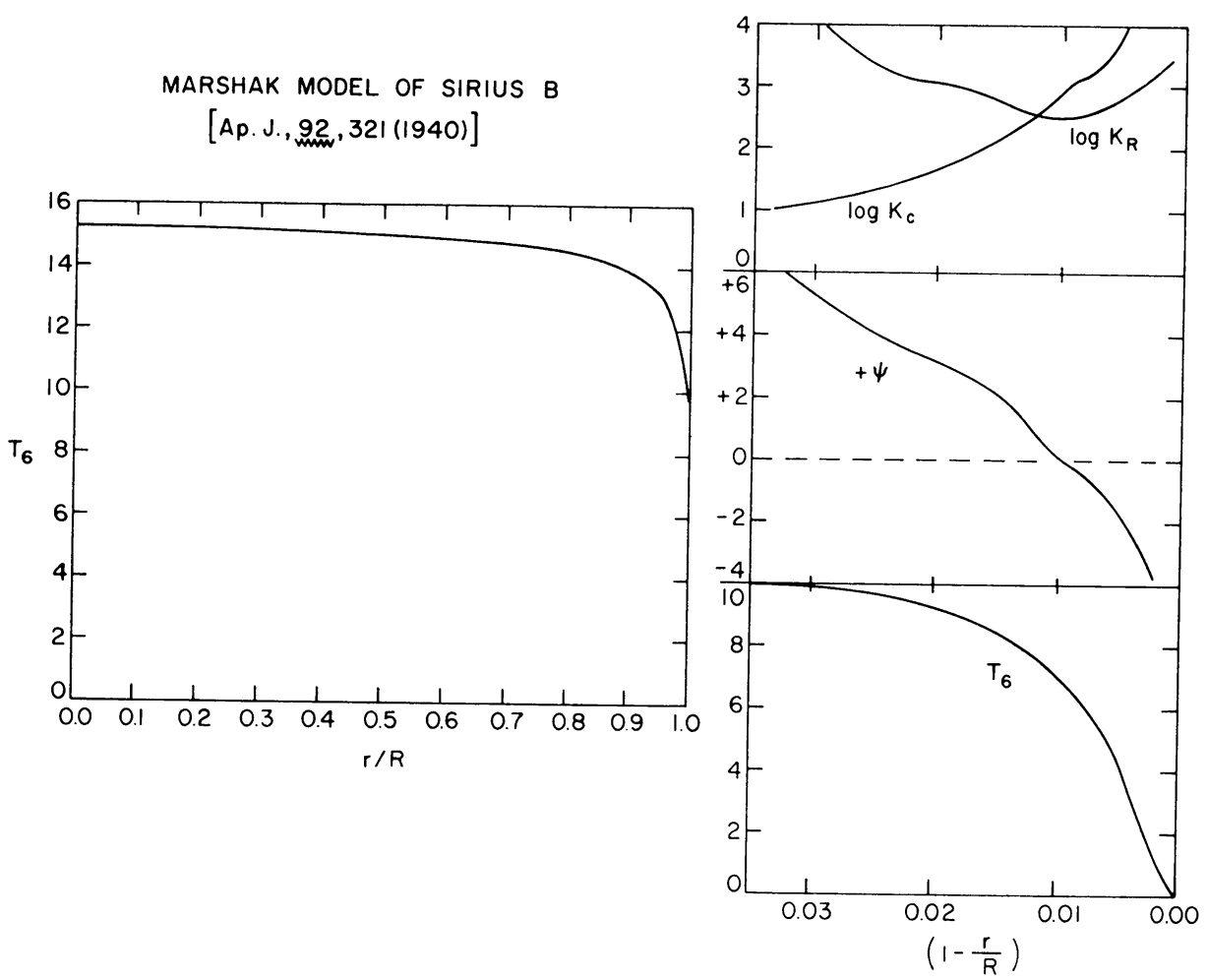

Fig. 5. Temperature distribution in Sirius B, as computed by Marshak (1940): $T_{6}=T / 10^{6} \mathrm{~K}$, and $R$ is the stellar radius. In the right-hand figure are plotted the distributions of temperature, of the degeneracy parameter $\psi$ ( $\psi k T$ is the Gibbs free energy per electron), and of the radiative, $K_{R}$, and conductive, $K_{C}$, opacities near the boundary of the degenerate core.

radiative and conductive opacities in order to carry out a precise computation of the temperature distribution and thermal energy content of a white dwarf. The assumption of a strictly isothermal core interior to the point where $\psi=0$ clearly leads to an underestimate of the total thermal energy and thus of the age by a factor of order two.

\section{A. CONDUCTIVE OPACITIES}

The high efficiency of heat conduction by the degenerate electrons in the interior of a white dwarf was established by Marshak (1940) and extended to the case of partial 
degeneracy by Mestel (1950) and Lee (1950). In this group of theories, conventionally termed the MML theory of the conductive opacity, the electrons are assumed to be non-relativistic and to be scattered only by the ions, which are taken to be infinitely massive point charges distributed at random through the plasma. While this process does in fact, provide the main contribution to the thermal resistivity, a number of other processes may also be significant in certain cases, as discussed by Abrikosov (1964) and by Hubbard (1966).

A substantial improvement in the treatment of electron conduction was made by Hubbard (1966), who carried out a rigorous calculation of the electron-ion scattering probability for a plasma and provided an approximate justification of the ad hoc cutoff procedure involved in the MML theory to avoid the divergence of the scattering cross-section associated with the long range of the coulomb force. Hubbard pointed out that the correlations among the positions of the ions induced by the Coulomb forces screens out the long-range electron-ion interactions, thus yielding a naturally convergent result. For typical white dwarf conditions Hubbard's opacities tend generally to be somewhat lower than corresponding MML opacities; the differences are of the order of a factor of two.

The effect of electron-electron scattering upon the conductivity has been studied intensively by Lampe (1968) and by Hubbard and Lampe (1969). These processes are normally neglected because the Pauli exclusion principle restricts the phase space available to both scattered electrons and thus inhibits these processes relative to electron-ion scattering. However, as Lampe (1968) has shown, near the core-envelope boundary, where the degeneracy is not strong, the electron-electron term can reduce the conductivity by $25-50 \%$. When this process is taken into account, as in the extensive tabulations of Hubbard and Lampe (1969), the resulting opacity values are found to agree extremely well with the MML results in the degenerate core (the opacity is increased above the value given by Hubbard), but is about a factor of two larger than the MML result in the non-degenerate region.

An important and badly needed extension of the Hubbard-Lampe theory to the conductivity of relativistic, semi-degenerate electrons has been published recently by Canuto (1970). At a density of $10^{6} \mathrm{~g} \cdot \mathrm{cm}^{-3}$, approximately the crossover from nonrelativistic to relativistic degeneracy, the agreement with the Hubbard-Lampe results is quite close.

Two other processes that have also been considered are scattering from impurities (Hubbard and Lampe, 1969) and from phonons (Solinger, 1970) both of which can take place in the solid phase. Neither of these processes contribute significantly to the conductivity in a white dwarf, however.

\section{B. RADIATIVE OPACITIES}

The most extensive tabulations of radiative opacity data for astrophysically interesting element mixtures are those published by Cox and Stewart (1965). These calculations include contributions from large numbers of absorption lines as well as from boundfree, free-free, and electron scattering transitions. Corrections for partial electron 
degeneracy and plasma screening of the ionic potentials are included. Recently, Carson et al. (1968) have also attacked the problem of opacity calculations, but by a rather different method, using the high temperature Thomas-Fermi-Dirac atom model. For the two element-mixtures they have treated in common with Cox and his collaborators they find close agreement in the limit of high ionization. For low ionization,

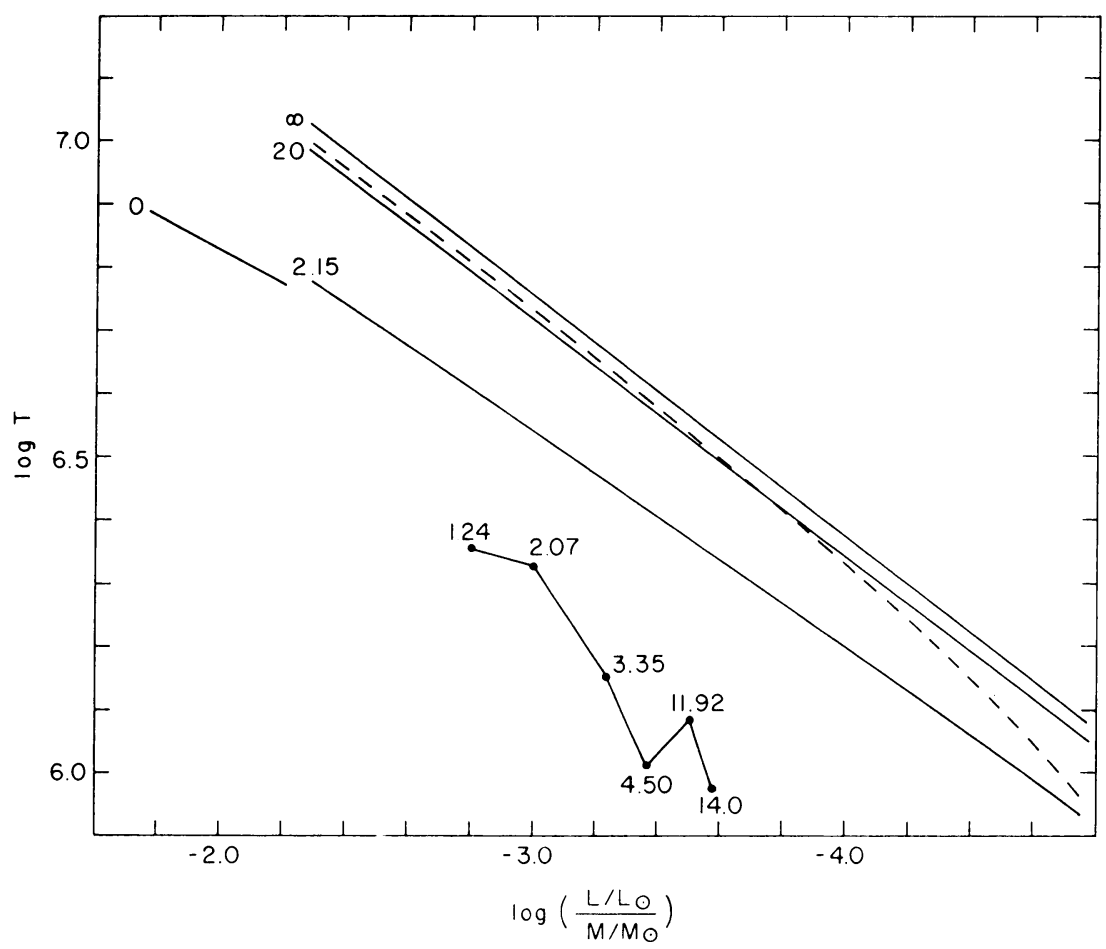

Fig. 6. Effects of different opacity laws and of envelope convection upon the core temperatures of white dwarfs. The solid curves show for helium envelopes the variation with $L / M$ of the temperature at different values of $\psi$ (the numbers attached to the curves indicate the appropriate $\psi$ values), as computed by Lacis (1970) for MML conductive opacities and Kramers' radiative opacities. The dashed curve, also due to Lacis, is the temperature at $\psi==20$ calculated with Hubbard-Lampe conductive opacities and Cox-Stewart radiative opacities. The temperature distribution at $\psi=0$, is for a set of helium envelope models that include convection, as computed by Van Horn (1970). The jointed curve gives the distribution of temperatures at the base of the convective zone, as computed by Böhm (1970); the numbers along the curve refer to the $\psi$ values at the bottom of the convective zone.

however, their opacities are larger by about a factor of two. This may be significant when envelope convection becomes important and the origin of the differences between these results should be investigated carefully.

Two additional effects that may become important in white dwarf envelopes are (i) the effect of plasma dispersion upon the Rosseland mean (Cox and Giuli, 1968) and (ii) the effect of the coulomb-induced ion correlations upon the processes of radiation 
absorption (Watson, 1970). Current estimates put both of these effects at about the ten percent level in typical white dwarf envelopes, although in certain cases they may be somewhat larger. More work on the difficult problem of opacity computations is thus desirable, but for purposes of establishing the relation between core temperature and luminosity the present calculations are probably adequate, at least for the white dwarfs of higher luminosity.

A detailed investigation of the effects on the $L-T_{c}$ relation of differences in the opacity laws has recently been carried out by Lacis (1970). The results of his study are briefly summarized in Figure 6, where the temperatures at degeneracies of $\psi=2.15$, 20 , and $\infty$ are plotted as functions of the luminosity-to-mass ratio that characterizes the envelopes. These calculations made use of Marshak's (1940) version of Kramers' radiative opacity law and of the MML conductive opacity. Also shown in this figure are the temperatures at $\psi=20$ in a model envelope computed using the Cox and Stewart (1965) radiative opacities for an almost pure helium mixture and the Hubbard and Lampe (1969) conductive opacities. The results agree to within about ten percent, indicating that present knowledge of the opacity laws is probably sufficient to provide a satisfactory determination of the $L-T_{c}$ relation. This conclusion differs from that expressed by Hubbard and Wagner (1970) for reasons outlined below.

\section{Envelope Convection in White Dwarfs}

Although it has been known for some time that the envelopes of white dwarfs should be convectively unstable (Schatzman, 1958, p. 47; Kolesov, 1964), it was not until recently that Böhm $(1968,1969)$ pointed out that the extensive convective zones of the cooler white dwarfs result in appreciably lower core temperatures than expected on the basis of the radiative envelope model. In Figure 6 we have plotted the temperatures, obtained for nearly pure helium envelopes by Böhm (1970), at the inner boundaries of the convective zones (labeled by the degeneracy parameters $\psi$ ) as a function of $L / M$. It is evident that the core temperatures are substantially reduced; the reduction is about a factor of 3 at the lowest luminosity considered. The jog in this curve is due to the fact that the models with lower luminosities have two convection zones, the outer zone corresponding to the region of hydrogen and first helium ionization, while the deeper zone coincides with that of second helium ionization.

The coincidence of convective regions with partial ionization zones is a general phenomenon arising from the combination of very high opacities and reduced adiabatic gradients caused by the incomplete ionization (Hayashi et al., 1962, p. 80). Convection is thus expected in the outer envelopes of all white dwarfs. This is confirmed by preliminary calculations for pure hydrogen, helium, and carbon envelope models (Van Horn, 1970). It is found that subsurface convection, beginning at the photosphere, occurs in all white dwarfs, but that the convective region occupies a smaller fraction of the envelope for the more luminous white dwarfs, while it may extend into the degenerate core at the lower luminosities. In the latter cases, the core temperature 
becomes quite sensitive to the actual atmospheric boundary conditions used; the models of Van Horn (1970) give much lower core temperatures in this luminosity range than the considerably more accurate models of Böhm (1970) which make use of actual atmospheric models computed by Grenfell and Böhm (1970). To obtain a reliable estimate of the $L-T_{c}$ relation at such low luminosities it is, therefore, very important to have accurate opacity laws and atmospheres.

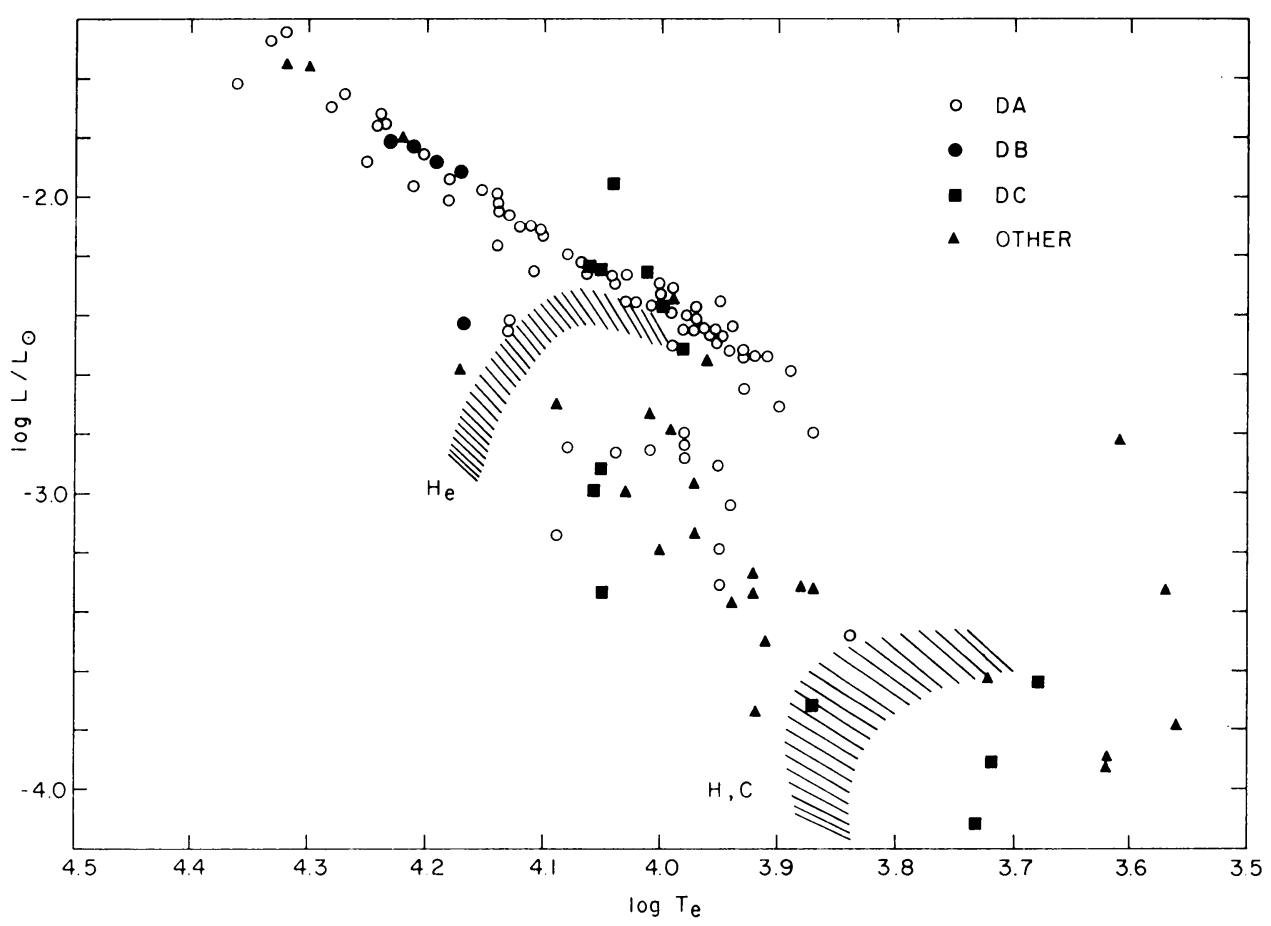

Fig. 7. Boundaries in the $\mathbf{H}-\mathbf{R}$ diagram of regions where envelope convection becomes important, shown together with the observations of Eggen and Greenstein (1965), with spectral types indicated. Below the upper hatched area marked ' $\mathrm{He}$ ' the convective zone in a helium envelope extends into the degenerate core. Similar results hold for the hydrogen and carbon convection boundaries, indicated by the lower hatched area marked ' $\mathrm{H}, \mathrm{C}$ '.

At the higher luminosities, where convection does not extend in to $\psi=0$, the temperature distribution near the core-envelope boundary is independent of the surface conditions. This is a consequence of the well-known tendency of envelope models to converge to the so-called 'radiative zero' solution (Schwarzschild, 1958, p. 92). The effect is shown graphically by Lacis (1970) and by Van Horn (1970) and is indicated in Figure 6 by the distribution of temperatures at $\psi=0$ obtained for helium envelopes with extensive convection zones in the outer parts. The good agreement of the temperatures of these models with results of the radiative envelope calculations thus indicates that prospects for obtaining accurate $L-T_{c}$ relations for the brighter white dwarfs are much better than anticipated by Hubbard and Wagner. 
Finally, it is of interest to ask in what regions of the $\mathrm{H}-\mathrm{R}$ diagram we may expect envelope convection to be important. This is qualitatively answered in Figure 7, where the loci of points defined by the condition that the inner boundary of the convective zone just reach the edge of the degenerate core are plotted for envelopes composed of hydrogen, of helium, and of carbon. It is interesting to note that no white dwarfs of spectral type DB (strong helium lines) lie below the He-convection boundary, and that no DA stars (strong hydrogen lines) fall below the edge of the $\mathrm{H}, \mathrm{C}$-zone. A refined theory of white dwarf envelope convection such as that being developed by Böhm and his coworkers may soon be able to tell us whether this result is real and significant.

\section{Acknowledgements}

This work has been supported in part by the National Science Foundation under grant GP-13695. Some of the numerical calculations were carried out at the Computing Center of the University of Rochester, which is in part supported by National Science Foundation grant GJ-828.

\section{References}

Abrikosov, A.: 1961, Soviet Phys.-JETP 12, 1254.

Abrikosov, A.: 1964, Soviet Phys.-JETP 18, 1339.

Adams, J. B., Ruderman, M. A., and Woo, H. C.: 1963, Phys. Rev. 129, 1383.

Beaudet, G., Petrosian, V., and Salpeter, E. E.: 1967, Astrophys. J. 150, 979.

Beaudet, G. and Salpeter, E. E.: 1969, Astrophys. J. 155, 203.

Böhm, K. H.: 1968, Astrophys. Space Sci. 2 , 375.

Böhm, K. H.: 1969, in Low Luminosity Stars (ed. by S. S. Kumar), Gordon and Breach, New York, p. 393.

Böhm, K. H.: 1970, Astrophys. J. 162, 919.

Brush, S. G., Sahlin, H. L., and Teller, E.: 1966, J. Chem. Phys. 45, 2102.

Canuoto, V.: 1970, Astrophys. J. 159, 641.

Carson, T. R., Mayers, D. F., and Stibbs, D. W. N.: 1968, Monthly Notices Roy. Astron. Soc. $140,483$. Cox, A. N. and Stewart, J. N.: 1965, Astrophys. J. Suppl. 11, 22.

Cox, J. P. and Giuli, T. T.: 1968, Principles of Stellar Structure, Gordon and Breach, New York, Chapter 8.

Eggen, O. J. and Greenstein, J. L.: 1965, Astrophys. J. 141, 83.

Feynman, R. P. and Gell-Mann, M.: 1958, Phys. Rev. 109, 193.

Grenfell, T. C. and Böhm, K. H.: 1970, Astrophys. J. 161, 1183.

Hayashi, C., Hoshi, R., and Sugimoto, D.: 1962, Suppl. Prog. Th. Phys., \# 22.

Hubbard, W. B.: 1966, Astrophys. J. 146, 858.

Hubbard, W. B. and Lampe, M.: 1969, Astrophys. J. Suppl. 18, 297.

Hubbard, W. B. and Wagner, R. L.: 1970, Astrophys. J. 159, 93.

Inman, C. L. and Ruderman, M. A.: 1964, Astrophys. J. 140, 1025.

Kirzhnits, D. A.: 1960, Soviet Phys.-JETP 11, 365.

Kolesov, A. K.: 1964, Soviet Astron.-AJ 8, 387.

Kutter, G. S. and Savedoff, M. P.: 1969, Astrophys. J. 157, 1021.

Lacis, A.: 1970, unpublished Ph.D. thesis, University of Iowa.

Lampe, M.: 1968, Phys. Rev. 170, 306.

Landau, L. D. and Lifshitz, E. M.: 1958, Statistical Physics, Addison-Wesley Publ. Co., Reading, Mass., p. 187.

Lawrence, G. M., Ostriker, J. P., and Hesser, J. E.: 1967, Astrophys. J. 148, L161.

Ledoux, P. J. and Sauvenier-Goffin, E.: 1950, Astrophys. J. 111, 611. 
Lee, T. D.: 1950, Astrophys. J. 111, 625.

Marshak, R. E.: 1940, Astrophys. J. 92, 321.

Mestel, L.: 1950, Proc. Camb. Phil. Soc. 46, 331.

Mestel, L.: 1952, Monthly Notices Roy. Astron. Soc. 112, 583.

Mestel, L.: 1965, in Stars and Stellar Systems 8 (ed. by L. H. Aller and D. B. McLaughlin), University of Chicago Press, Chicago, Chapter 5.

Mestel, L. and Ruderman, M. A.: 1967, Monthly Notices Roy. Astron. Soc. 136, 27.

Ostriker, J. P. and Axel, L.: 1969, in Low Luminosity Stars (ed. by S. S. Kumar), Gordon and Breach, New York, p. 357.

Salpeter, E. E.: 1961, Astrophys. J. 134, 669.

Savedoff, M. P., Van Horn, H. M., and Vila, S. C.: 1969, Astrophys. J. 155, 221.

Schatzman, E.: 1958, White Dwarfs, North-Holland Publ. Co., Amsterdam.

Schwarzschild, M.: 1958, Structure and Evolution of the Stars, Princeton University Press, Princeton.

Solinger, A.: 1970, paper presented at 132nd meeting Am. Astr. Soc.

Sudarshan, E. C. G. and Marshak, R. E.: 1958, Phys. Rev. 109, 1860.

Van Horn, H. M.: 1968, Astrophys. J. 151, 227.

Van Horn, H. M.: 1969, Phys. Letters 28A, 706.

Van Horn, H. M.: 1970, Astrophys. J. 160, L53.

Vila, S. C.: 1965, Ph.D. thesis, University of Rochester.

Vila, S. C.: 1966, Astrophys. J. 146, 437.

Vila, S. C.: 1967, Astrophys. J. 149, 613.

Watson, W. D.: 1970, Astrophys. J. 159, 641.

Weidemann, V.: 1968, Ann. Rev. Astron. Astrophys. 6, 351.

Zaidi, M. H.: 1965, Nuovo Cimento 40, 502. 